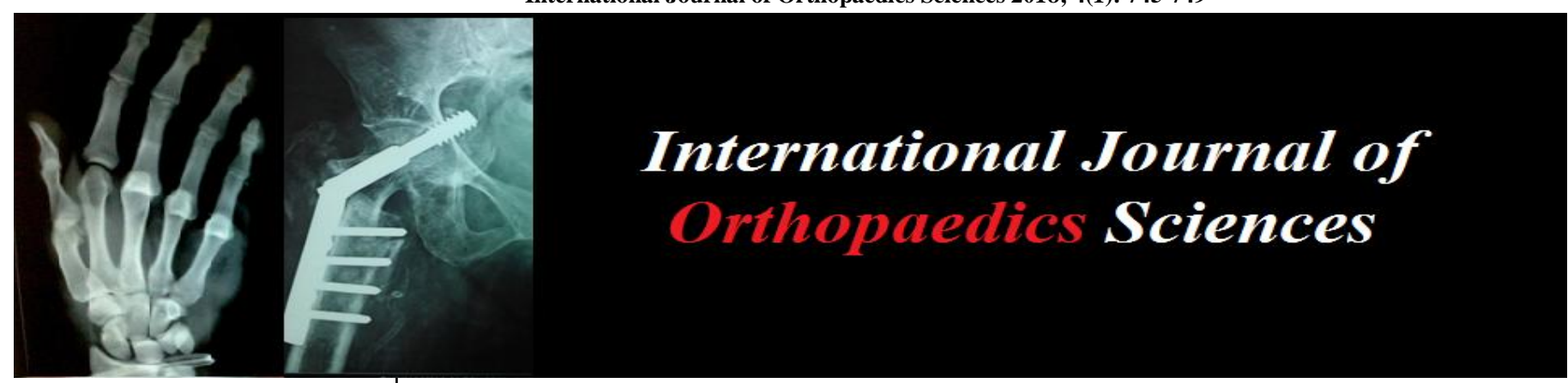

ISSN: $2395-1958$

IJOS 2018; 4(1): 745-749

(C) 2018 IJOS

www.orthopaper.com

Received: 28-11-2017

Accepted: 29-12-2017

Bishnu Pokharel

Department of Orthopaedics, BPKIHS, Dharan, Nepal

Rosan Prasad Shah Kalawar Department of Orthopaedics, BPKIHS, Dharan, Nepal

Guru Prasad Khanal Department of Orthopaedics, BPKIHS, Dharan, Nepal
Correspondence Misbah Mehraj

Assistant. Professor, Department of Orthopaedics, BPKIHS,

Dharan, Nepal

\section{Short term outcome of trans-portal anatomic single bundle anterior cruciate ligament reconstruction at BPKIHS}

\author{
Bishnu Pokharel, Rosan Prasad Shah Kalawar and Guru Prasad Khanal
}

DOI: $\underline{\text { https://doi.org/10.22271/ortho.2018.v4.i1k.108 }}$

\section{Abstract}

Introduction: Anterior cruciate ligament (ACL) reconstruction is performed to obtain pre-injury stability and to prevent early osteoarthritis of the knee in patients with ACL injury. This study was conducted to evaluate short term clinical and functional outcome of knee after trans-portal anatomic single bundle ACL reconstruction.

Methods: It was a prospective longitudinal study conducted in Department of Orthopedics, BPKIHS, Dharan, Nepal from Jan. 2017 to Sept.2017. All the patients with symptomatic ACL injury were included in the study. There were twenty patients who underwent trans-portal anatomic single bundle ACL reconstruction with quadrupled hamstring (Semitendinosus and gracilis) autograft from ipsilateral limb. The graft was fixed in femoral tunnel by an Endobutton CL and in tibia by Bioscrew. Results were assessed at 2 weeks, 6 weeks, 3 month and 6 months. Standard accelerated ACL rehabilitation protocol was followed for postoperative rehabilitation.

Results: Out of 20 patients who underwent ACL reconstruction surgery 16 were male and 4 female. Average graft size was $7 \pm 0.5 \mathrm{~mm}$. The longest follow up was 9 months and shortest follow up was 6 months. 18 patients could do active SLR at two weeks and 2 had extension lag of 10 degree. 19 out of 20 patients attained full range of movement at 6 weeks. Mean Preoperative Tegner Lysolm score was $63.852 \pm 1.19$ and post-operative Tegner Lysolm score at 6 months was $93 \pm 3.94$ with $\mathrm{P}$ value 0.0001 . There was no infection in any case.

Conclusion: Transportal anatomical single bundle ACL reconstruction with quadrupled hamstring autograph which is fixed with Endobutton on femur and intrafix bioscrew at tibia is very good method of ACL reconstruction with predictable outcome.

Keywords: Anterior cruciate ligament, outcome, short term, reconstruction, Tegner- Lysholm score

\section{Introduction}

Anterior cruciate ligament (ACL) injury is one of the most common injury of knee among the high level athletes which account for nearly $50 \%$ of total knee injury. It is also common in young and active non-sports people. Its prevalence is estimated to be 1 in 3000 in the United States (more than 120,000 cases annually) ${ }^{[1]}$. Anatomical ACL reconstruction has become the gold standard for the treatment of ACL tear to prevent instability and OA knee in long run. Ultimate goal of ACL reconstruction surgery is to return patients to his/her pre-injury status. Trans-portal approach for femoral tunnel preparation and placement of graft is widely used by arthroscopic surgeon with certain advantage over trans-tibial approach. Adoption of this approach has been shown improve femoral tunnel placement in terms of visualization, long horizontal tunnel placement and less chances for posterior blow out. Last but not the least it has obviate the need for notch plasty which is a regular procedure in transtibial approach [2].

The use of the semitendinosus and gracilis(STG) tendons is becoming the graft of choice in anterior cruciate ligament (ACL) reconstruction. The graft with four strands of STG tightened identically has a theoretical advantage over bone patellar tendon bone graft as its mechanical resistance is superior. There are no iatrogenic complications to extensor apparatus and no anterior knee pain.

A wide variety of fixation solutions to attach the hamstring tendons have been proposed. Most commonly used devices for femoral fixation are interference screws, transfix screws and 
cortical suspension devices. Devices for tibial fixation can be divided according to the location of fixation: intratunnel fixation and extratunnel fixation. Intratunnel fixation methods primarily rely on interference screw (metallic or bioabsorbable) or a cross pin system ${ }^{[3]}$.

Cortical suspension devices have been widely used in ACL reconstruction for femoral side graft fixation. Various studies have shown that cortical suspension devices have the necessary biomechanical properties with regard to ultimate failure strength, displacement, and stiffness for initial fixation of soft tissue in the femoral tunnel for ACL reconstruction ${ }^{\text {[4- }}$ ${ }^{6}$. Cortical suspension devices are available in two varieties 1. Fixed Loop-length device e.g. Endobutton and 2. Adjustable Loop-length device e.g. Tightrope. Endobutton is the first generation suspensory fixation with fixed-length loop. The length of the loop is fixed but it is stiffer and slippage-free which seems to have created a more favorable biomechanical environment.

The purpose of this study is to determine the functional outcome following anatomical single bundle ACL reconstruction using quadrupled hamstring autograft with femoral fixation of graft with Endobutton and tibial fixation with intrafix bioscrew.

\section{Material and methods}

It was a prospective longitudinal study conducted in department of Orthopedics, BPKIHS, Dharan, Nepal from Jan. 2017 to Sept. 2017. All the patients with symptomatic ACL injury which was confirmed by MRI were included in the study.

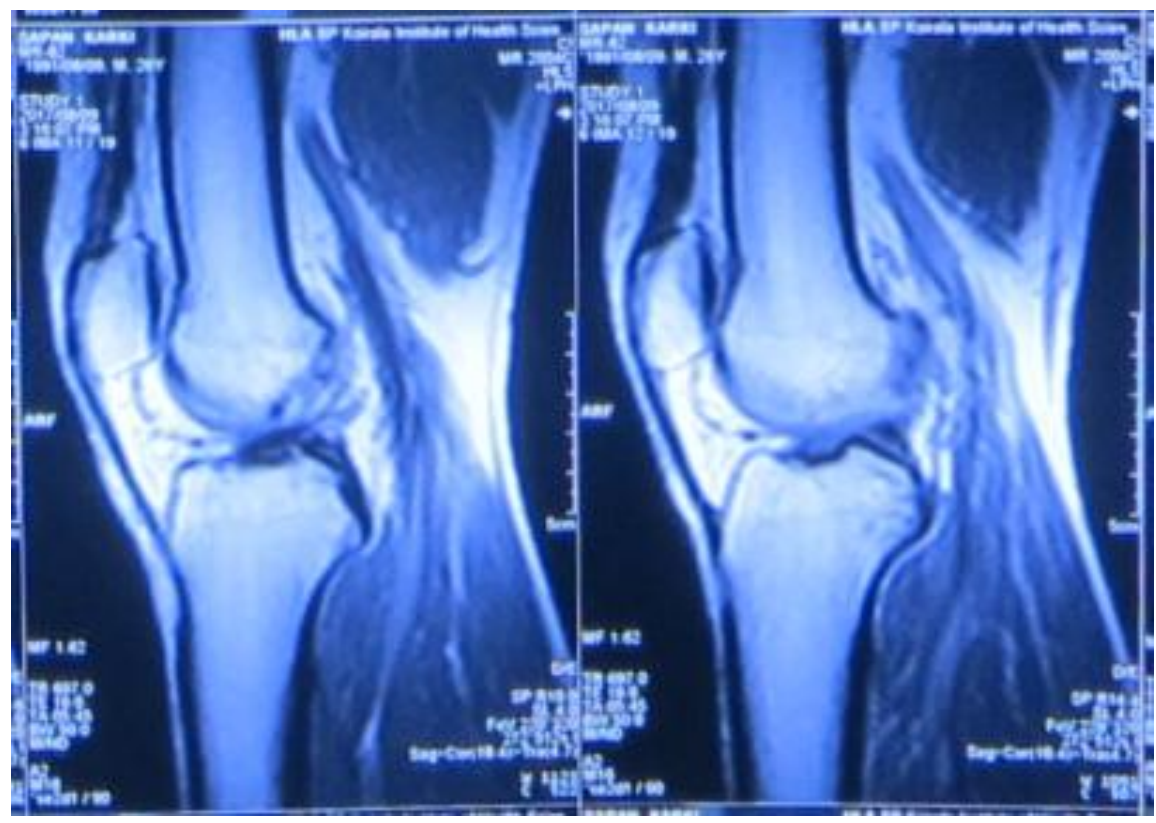

Fig 1: MRI of knee showing ACL rupture at femoral insertion site

Detail history regarding mode of injury, duration of injury and examination findings were noted. After adequate anesthesia, limb was assessed for Lachman and Pivot shift test.

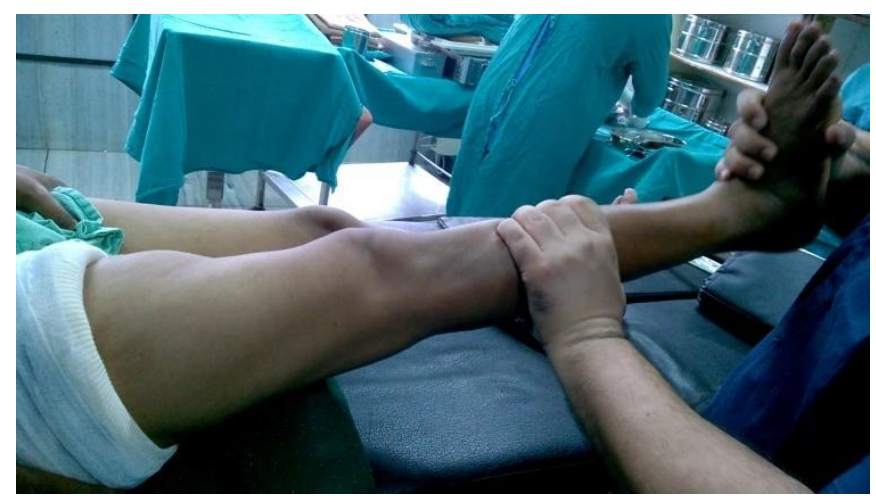

Fig 2: Peri-operative Pivot shift test

Each patient was put on Total Knee Replacement position and diagnostic arthroscopy was performed through two standard portals [Anterolateral (AL) and Anteromedial (AM)] and findings were noted (pattern of meniscus tear, type of chondral lesion). Then graft was harvested from the ipsilateral limb and prepared on ACL graft master. Femoral insertion of ACL was identified posterior to the median ridge and cleared with radiofrequency probe. Accessory anteromedial (AAM) portal was made just medial and inferior to AM portal and Beathpin was inserted under the guidance of Smith and Nephew femoral offset aimer hooked at posterior end of lateral femoral condyle knee flexed at 120 degree.

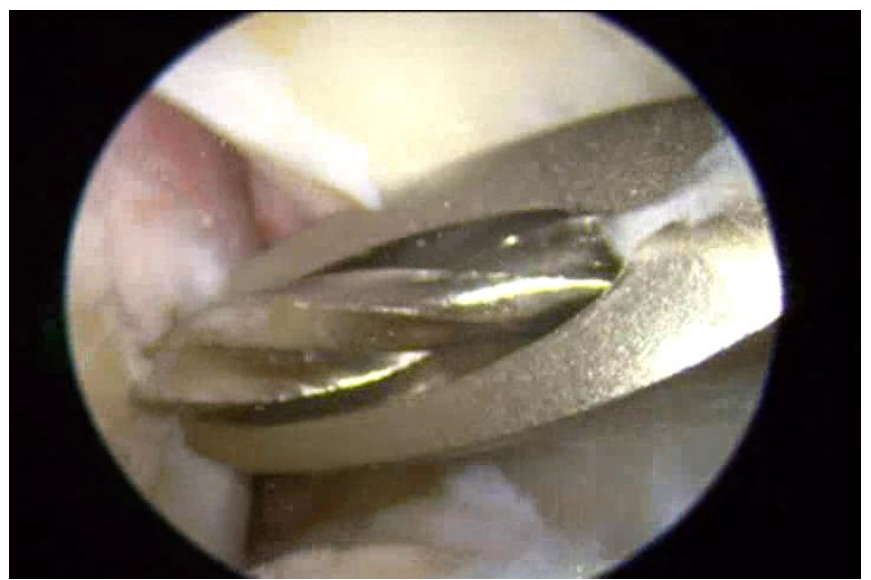

Fig 3: Femoral tunnel placement via femoral offset aimer

Beathpin was overdrilled with $4 \mathrm{~mm}$ cannulated drill bit, depth of the tunnel measured with depth gauze. After measurement of the tunnel, beathpin was inserted again and femoral tunnel was drill with endofermoral drill bit equal to the size of quadrupled hamstring graft keeping at least $5 \mathrm{~mm}$ 
of outer cortex intact. After drilling femoral tunnel, the tunnel is checked for posterior blow out and intactness of outer cortex directly by arthroscope.

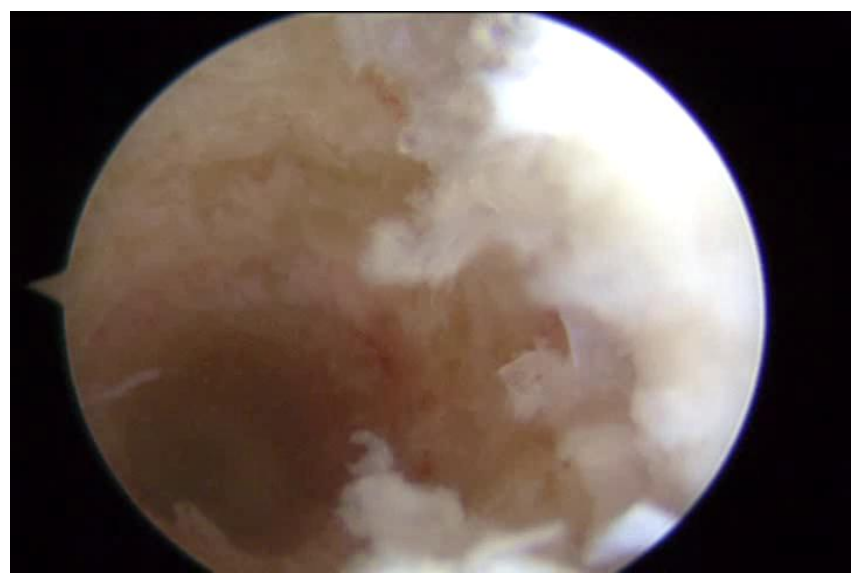

Fig 4: Femoral tunnel viewed through AAM portal

Then knee is extended and positioned at 90 degree of flexion. Tibial tunnel is made with the help of ACL tibial elbow aimer placed just anterior to the posterior border of anterior horn of lateral meniscus.

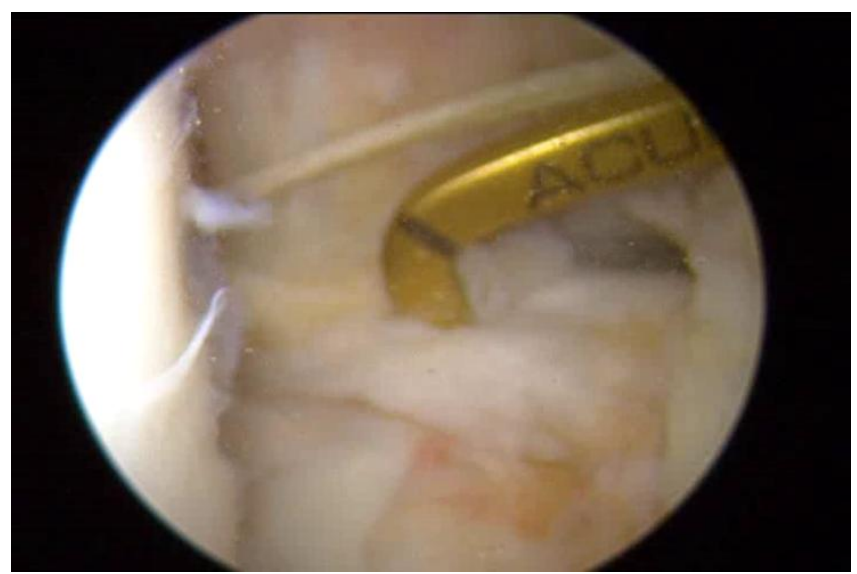

Fig 1: Tibial tunnel placement via tibial elbow aimer

The graft was looped in appropriate size Endobutton and inserted through tibial tunnel. The graft was first fixed in femoral tunnel by an Endobotton CL and graft tensioning was done by repeated flexion and extension of knee (15 to 20 cycles) with tibial end of graft held tightly on one hand. Then graft was fixed in tibia by Intrafix Bioscrew with knee flexed at 15 degree of flexion.

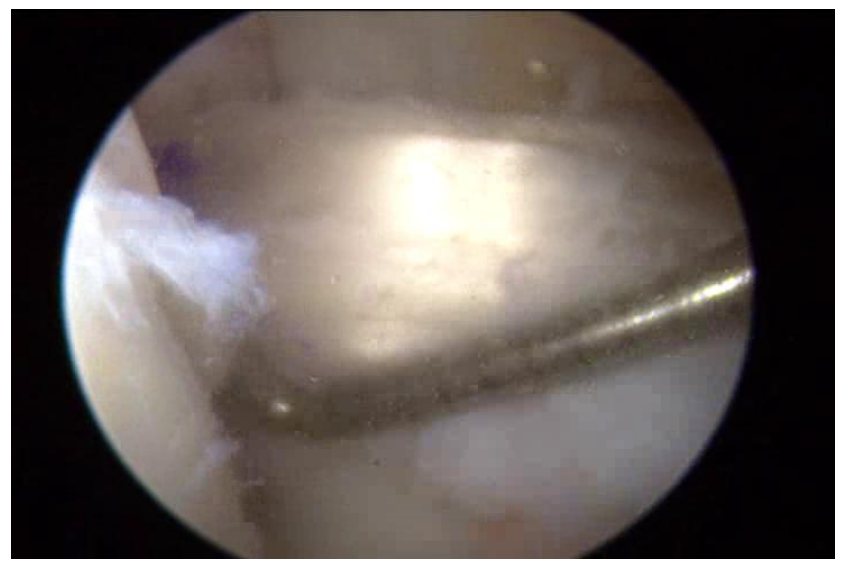

Fig 6: Graft after tibial side fixation

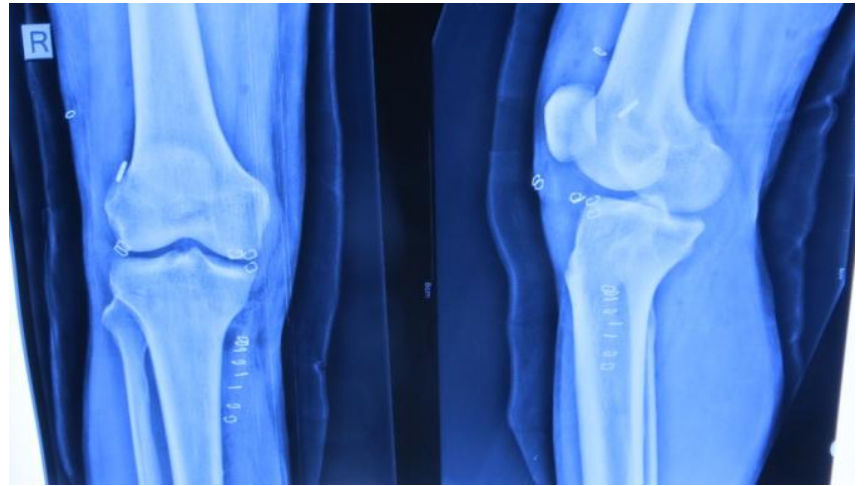

Fig 7: Post-operative check X-ray of knee

Standard structured ACL rehabilitation protocol was followed for postoperative rehabilitation. Wound dressing and discharge was done on $2^{\text {nd }}$ post-operative day and suture removal was done at 2 weeks. Patient assessed for infection, active SLR, extension lag, ROM, instability at 2 weeks, 6 weeks, 3 month and 6 months. Functional assessment of limb was performed by Tegner Lysolm Score one day prior to and at 6 months following surgery.

Data entry was done in Microsoft Excel 2010. Data were summarized in Table, charts, graph and diagram. Comparison of mean was done by Students t Test for normally distributed data.

\section{Results}

There were 27 patients who underwent ACL reconstruction during the study period. Seven patients were excluded from the study because they had less than 6 months of follow up. Out of the 20 patients only four were female, rest were male. Injury mostly occurred in the dominant limb right knee in 16 patients and left in rest. Age of the patients ranged from 18 years to 44 years with mean age $28.05 \pm 7.48$ years.

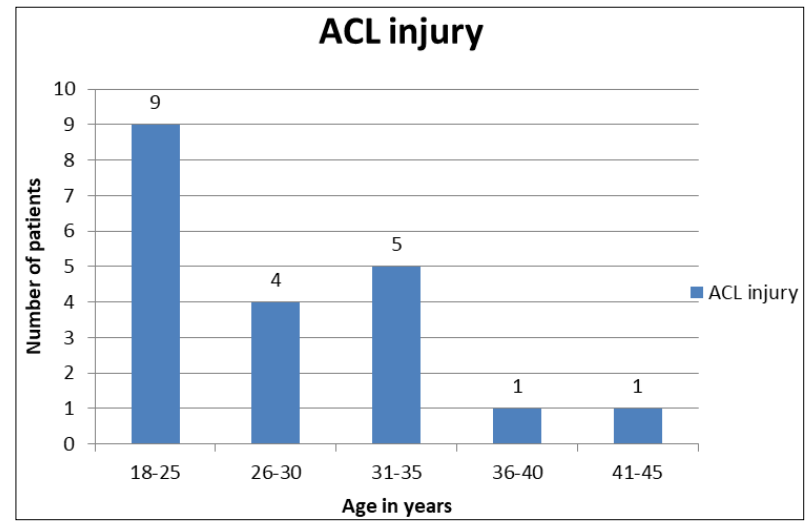

Fig 8: Age Distribution

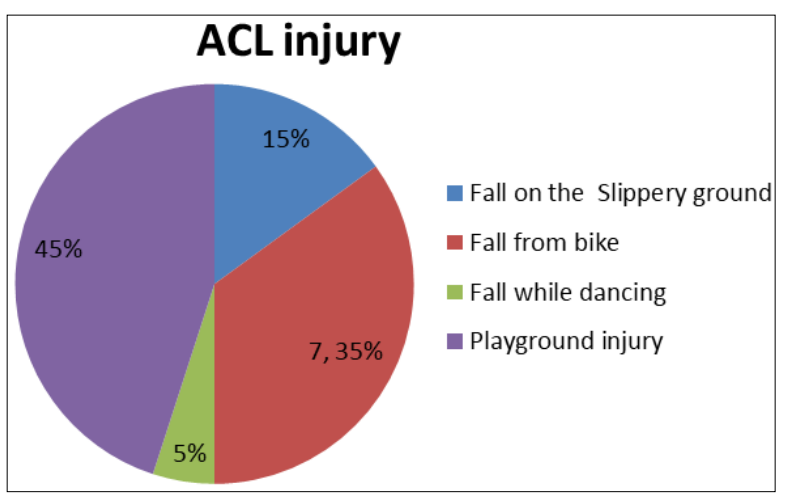

Fig 9: Mode of Injury 


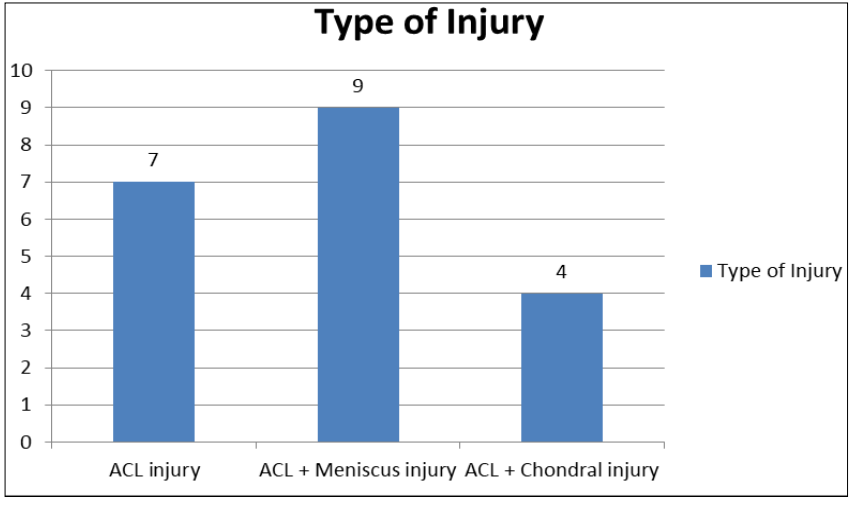

Fig 10: Operative findings

Seven patients had isolated ACL injury. Nine patients had associated meniscus injury out of which 7 had medial meniscus injury and 2 had lateral meniscus tear. 4 patients had chondral lesion on weight bearing area of tibia and femur.

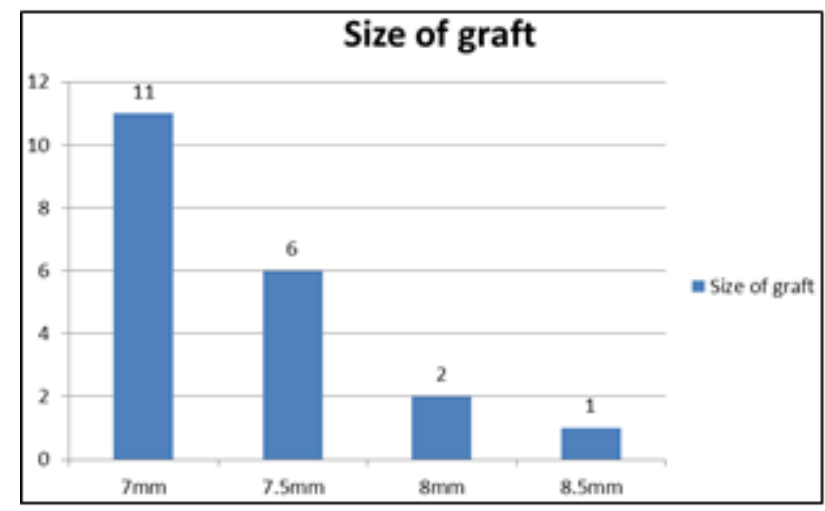

Fig 11: Size of quadruple hamstring autograft

Size of hamstring graft ranged from 7 to $8.5 \mathrm{~mm}$ with mean size of $7.325 \pm 0.181 \mathrm{~mm}$.

The longest follow up was 9 months and shortest follow up was 6 months. 18 patients could do active SLR at two weeks and 2 had extension lag of 10 degree. 19 out of 20 patients attained full range of movement at 6 weeks. One patient 40 years lady with grade 4 chondral lesion on weight bearing surface of medial femoral condyle took 3 months to attain full range of motion but extension lag still persisted.

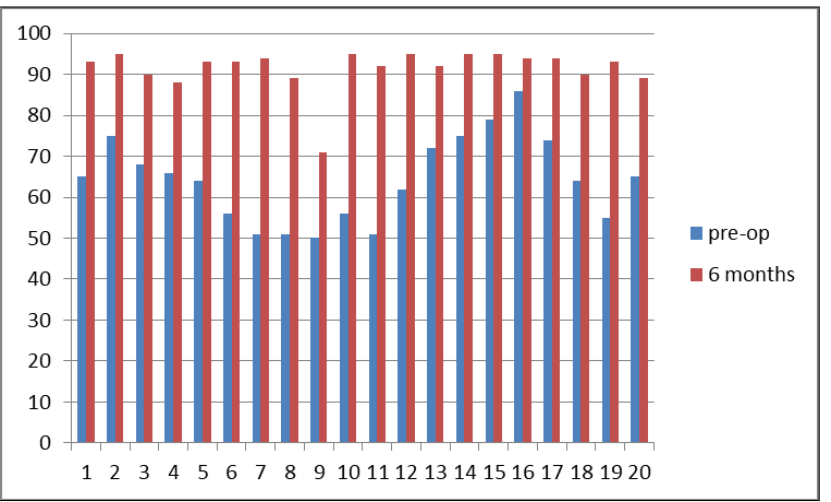

Fig 12: Pre-operative and post operative Tegner-Lysolm Scores

Mean Preoperative Tegner Lysolm score was 63.852 \pm 1.19 and post-operative Tegner Lysolm score at 6 months was $93 \pm 3.94$ with $\mathrm{P}$ value 0.0001 . Eight of them had excellent outcome and 12 of them had good outcome according to Tegner-Lysolm score. None of the patients developed infection following surgery.

\section{Discussion}

The concept of ACL reconstruction has been changing from non-anatomical transtibial to Transportal anatomical femoral tunnel placement of graft. Freddie Fu in one of his lecture told that, "When we do ACL surgery we should reconstruct it as anatomic as possible which is based on nature, form and function ${ }^{[7]}$." This concept has been widely accepted and practiced by most of the surgeons because anatomical ACL reconstruction has been found to closely restore the normal knee kinematics in patient with ACL deficient unstable knee. In our study we included 20 patients who underwent anatomic single bundle ACL reconstruction. Nine out of 20 patients fall in the age between 18 and 25 and majority of patients are under 40 years and male. People in this age group are socially active and involved in sports and recreation. So have the higher risk of injury which was further shown by the mode of injuries in our patients the most common playground injury followed by fall from bike.

Average size of the graft in our patient is $7 \mathrm{~mm}$ which is smaller than average graft size that found in other Asian male [8].

Conte EJ et.al in their systematic review found that ACL reconstruction with quadrupled- strand hamstring autograft with a diameter equal to or larger than $8 \mathrm{~mm}$ decreases failure rates ${ }^{[9]}$.

Mariscalco MW et al, in their multicentric retrospective chart review of prospectively collected cohort data of 263 patients undergoing primary ACL reconstruction with hamstring autograft, recorded graft size, femoral tunnel drilling technique, patient age, sex, and body mass index at the time of ACL reconstruction, Knee Injury and Osteoarthritis Outcome Score (KOOS) and International Knee Documentation Committee score preoperatively and at 2 years postoperatively; and whether each patient underwent revision ACL reconstruction during the 2-year follow-up period. They found that smaller hamstring autograft size $(8 \mathrm{~mm}$ or smaller) is a predictor of poorer KOOS sport/recreation function 2 years after primary ACL reconstruction ${ }^{[10]}$.

Since most of our patients are doing well at 6 months follow up, it is too early to predict the graft size and functional outcome of ACL reconstruction of our patients which need longer follow up.

Cortical suspension device has been one of the most widely used for femoral fixation of quadrupled hamstring graft in ACL reconstruction. These devices have ultimate failure strength greater than that necessary for early ACL rehabilitation for clinical use in ACL femoral fixation. It consists of a button that rests on the femoral cortex and a loop that hold the folded graft in position until healing occur. We used Endobutton for femoral fixation of graft. Functional status of ACL reconstructed limb in our patients showed significant improvement.

Similar study conducted by Kumar C et.al in 62 patients who underwent transportal anatomic ACL reconstruction with quadrupled hamstring graft fixed with Endobutton found good clinical outcome at short term follow up ${ }^{[11]}$.

Senthilkmar and Rajmohan conducted a prospective study of 15 cases of young injured ACL patients who underwent Arthroscopic Anatomical ACL reconstruction using single bundle Hamstring tendon with endobutton fixation in femoral side and interference screw in tibial side from April 2015 to July 2016. They used Tegner Lysolm score for functional assessment of the limb. They found that good to excellent results in paients with pure ACL injury where as poor result 
in patients with chondral injury ${ }^{[12]}$.

Similar study conducted by Mathur HH, et al. in 20 ACL injured patients treated by trans-portal anatomic single bundle anterior cruciate ligament reconstruction who were assessed by Modified Cincinnati Scoring System for preoperative ad post-operative function of injured limb. They found excellent result of the surgery without major complications ${ }^{[13]}$.

Our results are similar to above findings with significant difference in pre-operative and post-operative Tegner Lysolm Score with remarkable functional improvement of ACLR knee.

\section{Conclusion}

Anatomical single bundle ACL reconstruction with quadrupled hamstring autograph which is fixed with Endobutton on femur and intrafix bioscrew at tibia is very good method of ACL reconstruction with predictable outcome. There has been an encouraging result with these initial findings of ACL reconstruction. More number of patients with longer duration of follow up will be required to draw strong conclusion.

\section{References}

1. Kiapour AM, Murray MM. Basic sciences of Anterior cruciate ligament injury and repair. Bone Joint Res. 2014; 3:20-31. DOI: 10.1302/2046-3758.32.2000241

2. Yau WP, Fok AWM, Yee DKH. Tunnel Positions in Transportal Versus Transtibial Anterior Cruciate Ligament Reconstruction: A Case-Control Magnetic Resonance Imaging Study. Arthroscopy: The Journal of Arthroscopic \& Related Surgery. 2013; 29(6):1047-52. DOI: https://doi.org/10.1016/j.arthro.2013.02.010

3. Zeng C, Lei G, Gao S, Luo W. Methods and devices for graft fixation in anterior cruciate ligament reconstruction (Protocol). Cochrane Database of Systematic Reviews, 2013, 9. Art. No.: CD010730. DOI: 10.1002/14651858.CD010730.

4. Petre BM, Smith SD, Jansson KS, de Meijer PP, Hackett TR, LaPrade RF et al. Femoral cortical suspension devices for soft tissue anterior cruciate ligament reconstruction: a comparative study. Am J Sports Med. 2013; 41(1):416-22. $\quad$ PMID: 23263298 DOI: $10.1177 / 0363546512469875$

5. Johnson JS, Smith SD, LaPadre CM, Turnbull TL, LaPadre RF, Wijdicks CA. A biomechanical comparision of femoral cortical suspension devices for soft tissue anterior cruciate ligament reconstruction ubder high loads. Am J Sports Med. 2015; 43(1):154-60. PMID:25326014 DOI:10.1177/0363546514553779

6. More ADO, Pizzolatti ALA, Fancello EA, Roesler CRdM. ri Digiacomo Ocampo et al. Biomechanical performances of Bio Cross Pin and Endobutton for ACL reconstruction at femoral side: a porcine model. Res. Biomed Eng[online]. 2016; 36:28-34. DOI: http://dx.doi.org/10.1590/2446-4740.0720

7. Fu F. Anatomic ACL reconstruction, changing paradigm (Internet). Cited on: 1/30/2018 Available from: http://www.upmcphysicianresources.com/files/dmfile/An atomic-Acl-Reconstruction-9-14-11.pdf

8. Loo WL, Lui BYE, Lee YHD, Soon YHM. Can We Predict ACL Hamstring Graft Sizes in the Asian Male? A Clinical Relationship Study of Anthropometric Features and 4-Strand Hamstring Graft Sizes. Malaysian Orthopaedic Journal. 2010; 4(2):9-12.

9. Conte EJ, Hyatt AE, Gatt CJ, Dhawan A. Hamstring
Autograft Size Can Be Predicted and Is a Potential Risk Factor for Anterior Cruciate Ligament Reconstruction Failure. Arthoscopy. 2014; 3(7):882-90. DOI: http://dx.doi.org/10.1016/j.arthro.2014.03.028

10. Marscalo MW, Flanigan DC, Mitchell J, Pedroza AD, Jones MH, Andrish JT et al. The Influence of Hamstring Autograft Size on Patient Reported Outcomes and Risk of Revision After Anterior Cruciate Ligament Reconstruction: A Multicenter Orthopaedic Outcomes Network (MOON) Cohort Study. Arthroscopy: The Journal of Arthroscopic and Related Surgery. 2013; 29(12):1948-53. http://dx.doi.org/10.1016/j.arthro.2013.08.025

11. Kumar C, Gupta AK, Singh SK, Jain R. Transportal anterior cruciate ligament reconstruction with quadrupled hamstring tendon graft: A prospective outcome study. Ind J Orthop. 2017; 51(5):600-5.

12. Senthillkumar K, Rajmohan GA. Clinical outcome of Arthroscopic Anatomical Anterior cruciate ligament reconstruction using single bundle Hamstring tendon. IOSR Journal of Dental and Medical Sciences (IOSRJDMS. 2017; 16(9):01-04. DOI: 10.9790/08531609020104 www.iosrjournals.org

13. Mathur HH, Parmar, Tailor HK. Short term outcome of arthroscopic single bundle ACL reconstruction using quadruple hamstring graft by transportal technique for complete ACL tear. International Journal of Orthopaedics Sciences. 2017; 3(2):493-95. DOI: http://dx.doi.org/10.22271/ortho.2017.v3.i2e.43 